\title{
Adenosine 2A Receptor Occupancy by Tozadenant and Preladenant in Rhesus Monkeys
}

\author{
Olivier Barret ${ }^{1}$, Jonas Hannestad ${ }^{2}$, David Alagille ${ }^{1}$, Christine Vala ${ }^{1}$, Adriana Tavares ${ }^{1}$, Caroline Papin ${ }^{1}$, Thomas Morley ${ }^{1}$, \\ Krista Fowles ${ }^{3}$, Hsiaoju Lee ${ }^{1}$, John Seibyl ${ }^{1}$, Dominique Tytgat ${ }^{2}$, Marc Laruelle ${ }^{2}$, and Gilles Tamagnan ${ }^{1}$ \\ ${ }^{I}$ Molecular NeuroImaging, LLC, New Haven, Connecticut; ${ }^{2}$ UCB Pharma, Braine-l'Alleud, Belgium; and ${ }^{3}$ Yale PET Center, New \\ Haven, Connecticut
}

\begin{abstract}
Motor symptoms in Parkinson disease (PD) are caused by a loss of dopamine input from the substantia nigra to the striatum. Blockade of adenosine $2 A\left(A_{2 A}\right)$ receptors facilitates dopamine $D_{2}$ receptor function. In phase 2 clinical trials, $A_{2 A}$ antagonists (istradefylline, preladenant, and tozadenant) improved motor function in PD. We developed a new $\mathrm{A}_{2 \mathrm{~A}}$ PET radiotracer, ${ }^{18} \mathrm{~F}-\mathrm{MNI}-444$, and used it to investigate the relationship between plasma levels and $A_{2 A}$ occupancy by preladenant and tozadenant in nonhuman primates (NHP). Methods: A series of 20 PET experiments was conducted in 5 adult rhesus macaques. PET data were analyzed with both plasma-input (Logan graphical analysis) and reference-region-based (simplified reference tissue model and noninvasive Logan graphical analysis) methods. Whole-body PET images were acquired for radiation dosimetry estimates. Human pharmacokinetic parameters for tozadenant and preladenant were used to predict $A_{2 A}$ occupancy in humans, based on median effective concentration $\left(E_{50}\right)$ values estimated from the NHP PET measurements. Results: ${ }^{18} \mathrm{~F}-\mathrm{MNI}-444$ regional uptake was consistent with $A_{2 A}$ receptor distribution in the brain. Selectivity was demonstrated by dose-dependent blocking by tozadenant and preladenant. The specific-to-nonspecific ratio was superior to that of other $A_{2 A}$ PET radiotracers. Pharmacokinetic modeling predicted that tozadenant and preladenant may have different profiles of $A_{2 A}$ receptor occupancy in humans. Conclusion: ${ }^{18} \mathrm{~F}-\mathrm{MNI}-$ 444 appears to be a better PET radiotracer for $A_{2 A}$ imaging than currently available radiotracers. Assuming that $\mathrm{EC}_{50}$ in humans is similar to that in NHP, it appears that tozadenant will provide a more sustained $A_{2 A}$ receptor occupancy than preladenant in humans at clinically tested doses.
\end{abstract}

Key Words: $A_{2 A}$ receptors PET imaging; Parkinson's disease; receptor occupancy; tozadenant; preladenant

J Nucl Med 2014; 55:1712-1718

DOI: 10.2967/jnumed.114.142067

$\mathbf{P}$

arkinson disease (PD) has a prevalence of $1.6 \%$ in individuals over the age of $65 \mathrm{y}(1)$ and a lifetime risk of $6.7 \%$ from age 45 to 100 y (2). Motor symptoms, which include tremor, bradykinesia, and rigidity, emerge when there is a loss of more than $50 \%$ of dopamine neurons in the substantia nigra $(\mathrm{SN})(3,4)$. Loss of these

Received Apr. 22, 2014; revision accepted Jun. 27, 2014.

For correspondence or reprints contact: Olivier Barret, Molecular Neurolmaging

(MNI), LLC, 60 Temple St., Suite 8B, New Haven, CT 06510.

E-mail: obarret@mnimaging.com

Published online Jul. 31, 2014.

COPYRIGHT (c) 2014 by the Society of Nuclear Medicine and Molecular Imaging, Inc. neurons reduces dopamine input to the striatum, where dopamine binds to both $D_{1}$ and $D_{2}$ receptors. Most striatal $D_{1}$ receptors are localized in the so-called direct pathway, whereas most striatal $\mathrm{D}_{2}$ receptors are localized in medium spiny neurons that project to the globus pallidus pars externa (indirect pathway). Adenosine signals via 4 different G-protein-coupled receptors: $A_{1}, A_{2 A}, A_{2 B}$, and $A_{3}$ (5). $A_{2 A}$ receptors are predominantly expressed in striatum, with lower levels present in cortex and thalamus and even lower in cerebellum (5-9). $\mathrm{A}_{2 \mathrm{~A}}$ receptors may play a role in inflammation (10) and could therefore be important in a variety of neurologic diseases, including multiple sclerosis, in which $\mathrm{A}_{2 \mathrm{~A}}$ receptor density is increased (11). In PD, $\mathrm{A}_{2 \mathrm{~A}}$ receptors may be important because they form heterodimers with $\mathrm{D}_{2}$ receptors in the striatum $(5,12)$, and agonists of $\mathrm{A}_{2 \mathrm{~A}}$ (e.g., adenosine) reduce the affinity of $\mathrm{D}_{2}$ for dopamine (13). Therefore, antagonism of $\mathrm{A}_{2 \mathrm{~A}}$ receptors will facilitate $\mathrm{D}_{2}$ transmission, which should be beneficial in PD. Three $\mathrm{A}_{2 \mathrm{~A}}$ antagonists have been tested in PD: istradefylline, preladenant, and tozadenant. All 3 showed efficacy on motor symptoms in phase II trials (14-16); however, istradefylline was not approved in Europe or the United States because of mixed results in phase III trials. Recently, approval was granted in Japan (17). More recently, preladenant failed to demonstrate efficacy in phase III trials.

One important use of PET imaging is to quantify receptor occupancy $(\mathrm{RO})$ by a drug. Several PET radiotracers for $\mathrm{A}_{2 \mathrm{~A}}$ have been developed and tested $(5,6,8,18-20)$. The best characterized radiotracer, ${ }^{11} \mathrm{C}-\mathrm{SCH} 442416$, has a good maximum striatum-tocerebellum ratio in rodents $(\sim 5.0)$, but this ratio is lower in primates $(\sim 2.2)(5)$, thus limiting the dynamic range of the tracer for RO studies. We recently developed a new $A_{2 A}$ radiotracer for SPECT imaging for which striatum-to-cerebellum ratios of about 3.0-3.5 were measured in monkey and baboon brain (21). Subsequently, we labeled this molecule with ${ }^{18} \mathrm{~F}$ for use as a PET tracer. The objective of this study was to characterize this new $\mathrm{A}_{2 \mathrm{~A}}$ PET radiotracer, ${ }^{18} \mathrm{~F}-\mathrm{MNI}-444$, in vivo in nonhuman primates, including test-retest, dosimetry, and blocking studies with tozadenant and preladenant. In addition, we decided to perform pharmacokinetic modeling to try to understand whether suboptimal occupancy in humans might explain the lack of efficacy of preladenant in phase III trials.

\section{MATERIALS AND METHODS}

\section{Radiochemistry}

${ }^{18} \mathrm{~F}-\mathrm{MNI}-444$ was prepared by reaction of the corresponding tosyl precursor, 2-(4-(4-(2-(5-amino-2-(furan-2-yl)-7H-pyrazolo[4,3-e] $[1,2,4]$ triazolo[1,5-c]pyrimidin-7-yl)ethyl)piperazin-1-yl)phenoxy)ethyl4-methylbenzenesulfonate, with ${ }^{18} \mathrm{~F}^{-}$in anhydrous dimethylsulfoxide in the presence of potassium carbonate and Kryptofix 222 (Merck) at 
$100^{\circ} \mathrm{C}$ for 12 min using a commercial synthesizer, TRACERlab FX-FN (GE Healthcare) (Fig. 1). The resulting radiolabeled product was purified by semipreparative high-performance liquid chromatography with an Eclipse XDB C-18 column $(5 \mu \mathrm{m}, 10 \times 250 \mathrm{~mm}$; Agilent), eluted with a mobile phase of acetonitrile/water/triethylamine $(35: 65: 0.2 \mathrm{v} / \mathrm{v} / \mathrm{v})$ at a flow rate of $4 \mathrm{~mL} / \mathrm{min}$, and formulated in a physiologic solution containing ethanol, ascorbic acid, and polysorbate-80 in normal saline. Quality control showed radiochemical purity above $99 \%$ and a specific activity exceeding $370 \mathrm{GBq} / \mu \mathrm{mol}$ for all the productions (the supplemental data provide details, available at http://jnm.snmjournals.org). The average decay-corrected radiochemical yield was $24.5 \% \pm 5.0 \%(n=39)$ in $60 \mathrm{~min}$.

\section{Blocking Agent Preparation}

Tozadenant and preladenant are potent, selective, and structurally unrelated $\mathrm{A}_{2 \mathrm{~A}}$ antagonists. In vitro, tozadenant has a $\mathrm{K}_{\mathrm{i}}$ of $11.5 \mathrm{nM}$ on human $\mathrm{A}_{2 \mathrm{~A}}$ and $6 \mathrm{nM}$ on rhesus $\mathrm{A}_{2 \mathrm{~A}}$ whereas preladenant has an inhibition constant $\left(\mathrm{K}_{\mathrm{i}}\right)$ of $1.1 \mathrm{nM}$ on human $\mathrm{A}_{2 \mathrm{~A}}$. Tozadenant was provided by Biotie Therapies Corp. and dissolved in sterile water with $20 \%$ Captisol ( $\beta$-cyclodextrin sulfobutylether sodium; CyDex Pharmaceuticals). Preladenant was synthesized at Molecular NeuroImaging, LLC, and dissolved in normal saline at $\mathrm{pH} 3.0$ with $20 \%$ hydroxypropyl- $\beta$-cyclodextrin. RO experiments were performed with 6 doses of tozadenant $(0.5,1.3,1.5,5.0,7.5$, and $10.5 \mathrm{mg} / \mathrm{kg})$ and 8 doses of preladenant (0.004 in duplicate, 0.010, 0.015 in duplicate, 0.04, 0.2, and $1.0 \mathrm{mg} / \mathrm{kg}$ ) administered intravenously over $10 \mathrm{~min}$, through the same canula used for the radiotracer administration occurring 20 min later. Plasma samples were taken at several time points during each PET scan to estimate the relationship between RO and plasma exposure.

\section{${ }^{18}$ F-MNI-444 Brain PET Studies}

PET scans $(n=20)$ were obtained on a Focus 220 microPET camera (Siemens Healthcare Molecular Imaging) in adult rhesus macaques (Macaca mulatta, 2 female and 3 male, $7.8 \pm 2.9 \mathrm{~kg}$ ): 6 baseline studies ( 2 test-retest studies), 6 preblocking studies with tozadenant, and 8 preblocking studies with preladenant. PET images were acquired during $3 \mathrm{~h}$ after intravenous administration of $179.8 \pm 9.9$ $\mathrm{MBq}(0.6 \pm 0.4 \mu \mathrm{g})$ of ${ }^{18} \mathrm{~F}-\mathrm{MNI}-444$ over $3 \mathrm{~min}$ with an infusion pump. A transmission scan with an external ${ }^{68} \mathrm{Ge}$ source was performed before the emission scans. Images were reconstructed using

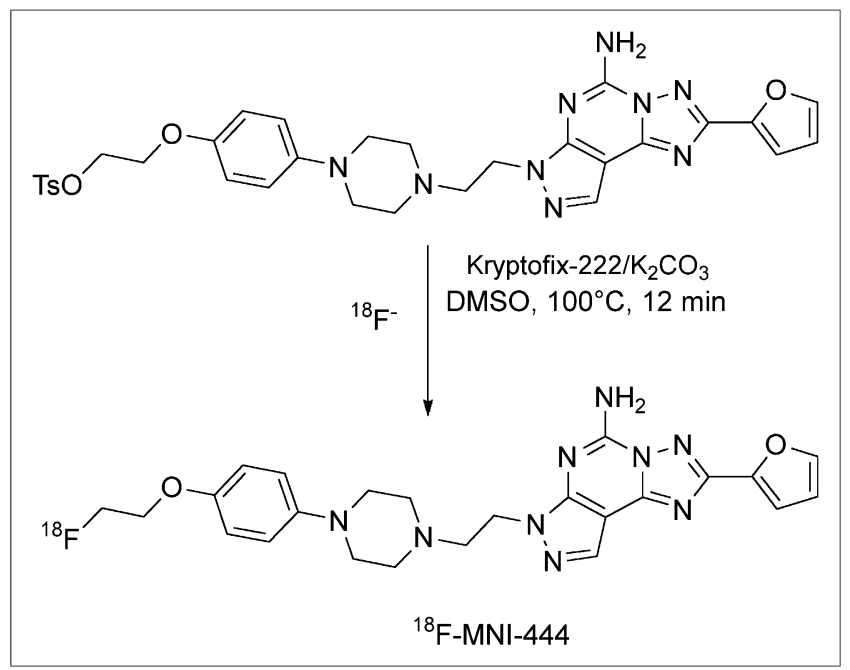

FIGURE 1. Radiosynthesis of ${ }^{18} \mathrm{~F}-\mathrm{MNI}-444$ from its tosylate precursor. DMSO = dimethyl sulfoxide. filtered backprojection with standard corrections for randoms, scatter, and attenuation.

After tracer administration, radial artery blood samples were collected over $3 \mathrm{~h}$. Radioactivity in whole blood and plasma was measured in all samples, and radiometabolites were measured in a subset. Plasma protein binding free fraction $\left(f_{\mathrm{p}}\right)$ was measured by ultrafiltration. The arterial plasma input function corrected for radiometabolites was used for the PET data analysis (supplemental data).

PET images were analyzed in PMOD, version 3.4 (PMOD Technologies). Initial flowlike PET images $(15 \mathrm{~min})$ were averaged and aligned onto the rhesus T1-weighted MR images and the transformation applied to the whole PET series. Volumes of interest, including the caudate, putamen, globus pallidus, nucleus accumbens, cortical regions, and cerebellum, were applied to the PET series to extract the time-activity curves. Curves were expressed in standardized uptake value by normalizing by the injected dose and animal body weight.

Time-activity curves were analyzed with Logan graphical analysis (LGA) (22) with $t^{*}$ set to $60 \mathrm{~min}$ to derive the volume of distribution in each region. The nondisplaceable binding potential $\left(\mathrm{BP}_{\mathrm{ND}}\right)$ was estimated using the cerebellum as the reference region: $\mathrm{BP}_{\mathrm{ND}}=$ $\mathrm{V}_{\mathrm{T}} / \mathrm{V}_{\mathrm{ND}}-1, \mathrm{~V}_{\mathrm{T}}$ and $\mathrm{V}_{\mathrm{ND}}$ being the distribution volumes in the target region and reference region (nondisplaceable uptake), respectively (23). In addition, $\mathrm{BP}_{\mathrm{ND}}$ was directly derived from both the simplified reference tissue model (SRTM) (24) and the noninvasive LGA (NI-LGA) (22) with $t^{*}$ set to $60 \mathrm{~min}$ and $k_{2}{ }^{\prime}$ set to $0.35 \mathrm{~min}^{-1}$. In vitro autoradiography experiments have shown that the cerebellum has low to negligible $A_{2 \mathrm{~A}}$ receptor density (9), and previous studies with other $\mathrm{A}_{2 \mathrm{~A}}$ radiotracers have used the cerebellum for estimation of tissue ratios and $\mathrm{BP}_{\mathrm{ND}}(5,8) . \mathrm{V}_{\mathrm{T}}$ and $\mathrm{BP}_{\mathrm{ND}}$ were obtained using either 180 or $120 \mathrm{~min}$ of data. Test-retest reproducibility for $\mathrm{V}_{\mathrm{T}}$ and $\mathrm{BP}_{\mathrm{ND}}$ was estimated in 2 animals as absolute value(test - retest)/average(test + retest).

The $\mathrm{A}_{2 \mathrm{~A}} \mathrm{RO}$ produced by tozadenant or preladenant was determined as the percentage change of $\mathrm{BP}_{\mathrm{ND}}$ :

$$
\operatorname{Occupancy}(\%)=\left(\mathrm{BP}_{\mathrm{ND}}^{\text {baseline }}-\mathrm{BP}_{\mathrm{ND}}^{\mathrm{drug}}\right) / \mathrm{BP}_{\mathrm{ND}}^{\text {baseline }} \times 100 . \quad \text { Eq. } 1
$$

The dose-occupancy curves for the striatum (putamen and caudate) were fitted in Prism, version 6.01 (GraphPad Software), with a single specific binding site model:

$$
\operatorname{Occupancy}(\%)=\mathrm{O}_{\max } \times \mathrm{D} /\left(\mathrm{D}+\mathrm{ED}_{50}\right) \text {, }
$$

where $\mathrm{O}_{\max }$ is the maximum occupancy, $\mathrm{ED}_{50}$ the drug dose for $50 \%$ occupancy, and D the drug dose. A similar fit was done against the plasma levels to determine the median effective concentration $\left(\mathrm{EC}_{50}\right)$.

\section{Human Pharmacokinetic Modeling and RO Estimates}

Pharmacokinetic data for tozadenant from phase I and II studies were incorporated into a population pharmacokinetic model to predict the median and fifth and 95th percentiles of tozadenant plasma levels achieved with $180 \mathrm{mg}$ twice a day (BID) in humans. For preladenant, pharmacokinetic parameters from a published phase I study (25) were used to predict steady-state plasma levels achieved with $10 \mathrm{mg}$ BID in humans. Differences in plasma free fraction between nonhuman primates (NHPs) and humans were accounted for to estimate the $\mathrm{EC}_{50}$ in humans:

$$
\text { Human } f_{\mathrm{p}} \cdot \text { human } \mathrm{EC}_{50}=\operatorname{NHP} f_{\mathrm{p}} \cdot \operatorname{NHP} \mathrm{EC}_{50} \text {. }
$$

Eq. 3

The $f_{\mathrm{p}}$ of preladenant is 0.03 and 0.01 in NHPs and humans, respectively (UCB Pharma data) (25). The $f_{\mathrm{p}}$ of tozadenant is 0.5 and 0.3 in NHPs and humans, respectively (UCB Pharma data). The free-fraction-corrected 


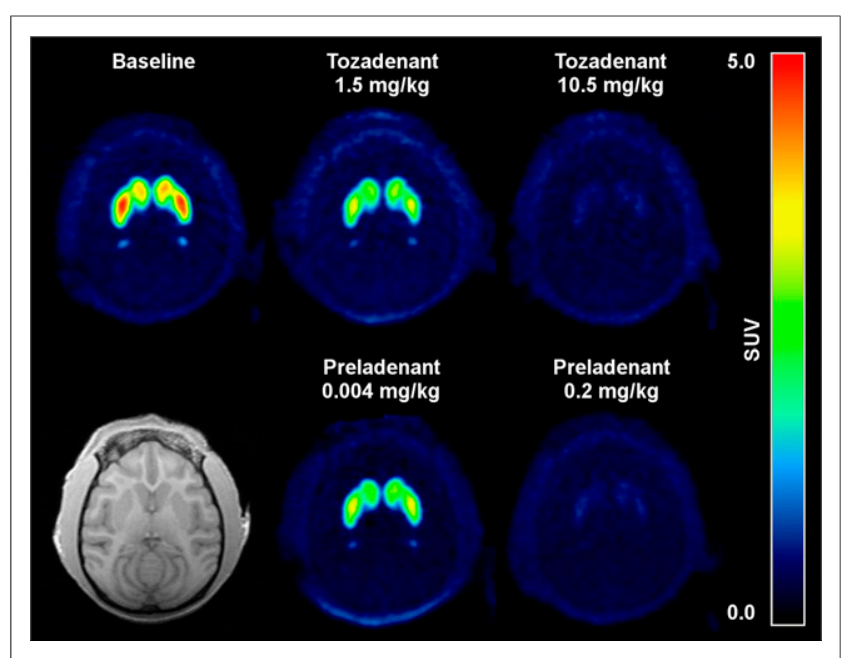

FIGURE 2. Average ${ }^{18} \mathrm{~F}-\mathrm{MNI}-444$ PET images over $180 \mathrm{~min}$ for rhesus macaque in transverse plane at baseline and after dosing with tozadenant at 1.5 and $10.5 \mathrm{mg} / \mathrm{kg}$ (occupancy of $47 \%$ and $95 \%$, respectively) or with preladenant at 0.004 and $0.2 \mathrm{mg} / \mathrm{kg}$ (occupancy of $32 \%$ and $90 \%$, respectively). Monkey individual MR image is also shown. SUV = standardized uptake value.

$\mathrm{EC}_{50}$ in humans was combined with the human pharmacokinetic model to estimate the $\mathrm{A}_{2 \mathrm{~A}}$ occupancy achieved with $180 \mathrm{mg}$ BID of tozadenant and $10 \mathrm{mg}$ BID of preladenant.

\section{F-MNI-444 Whole-Body PET Studies}

Two adult rhesus monkeys, 1 male and 1 female, were used for whole-body PET imaging to determine the biodistribution and estimate the absorbed radiation doses of ${ }^{18} \mathrm{~F}-\mathrm{MNI}-444$. The supplemental data provide details on the dosimetry methods.

\section{RESULTS}

\section{Plasma Analysis}

High-performance liquid chromatography analysis of arterial plasma revealed one major radiometabolite that was more polar than the parent compound and a second metabolite eluting just after the first one but whose contribution was less than $3 \%$ at any measured time point. Because of the polar nature of both of these metabolites, it is unlikely that either would readily enter the brain. ${ }^{18} \mathrm{~F}-\mathrm{MNI}-444$ was moderately metabolized, with about $45 \%$ and $10 \%-15 \%$ of intact parent remaining at $30 \mathrm{~min}$ and 120 min after injection, respectively (Supplemental Fig. 1).
Plasma parent $f_{\mathrm{p}}$ measured by ultrafiltration was $1.2 \% \pm 0.5 \%$ $(n=20)$.

\section{Brain PET Studies}

Representative average ${ }^{18} \mathrm{~F}-\mathrm{MNI}-444$ PET images showed high uptake in the striatum, consistent with $\mathrm{A}_{2 \mathrm{~A}}$ receptor density (Fig. 2) $(5,7) .{ }^{18} \mathrm{~F}-\mathrm{MNI}-444$ readily entered the brain, with a high peak standardized uptake value (30-60 min after injection) in the putamen, caudate, nucleus accumbens, and globus pallidus (Fig. 3A). In all other brain regions the uptake was lower and had a much faster washout, in particular for the cerebellum, with a peak uptake within 5 min of injection, consistent with much lower $\mathrm{A}_{2 \mathrm{~A}}$ receptor density in these regions $(5,7)$. No skull update was observed, suggesting no defluorination of this radiotracer.

Logan plots ( $t^{*}=60 \mathrm{~min}$, LGA and NI-LGA) and SRTM fits are provided in Figures 3A-3C for a representative baseline scan. SRTM determined $\mathrm{k}_{2}^{\prime}$ to be $0.37 \pm 0.15 \mathrm{~min}^{-1}$ and $0.34 \pm$ $0.13 \mathrm{~min}^{-1}$ for 180- and 120-min acquisitions, respectively, with improved fits for shorter acquisitions. For NI-LGA, $\mathrm{k}_{2}{ }_{2}$ was set to $0.35 \mathrm{~min}^{-1}$. For baseline scans $(n=6), \mathrm{LGA} \mathrm{V}_{\mathrm{T}}(180 \mathrm{~min})$ estimates ranged from $3.47 \pm 0.65$ in the cerebellum to $30.25 \pm 5.82$ in the putamen, with the rank order being putamen $>$ caudate $>$ globus pallidus $>$ nucleus accumbens $>>$ cortices $>$ cerebellum (Table 1). The $\mathrm{BP}_{\mathrm{ND}}$ in the putamen was estimated to be $8.03 \pm$ 2.66 with LGA, $9.64 \pm 1.94$ with SRTM, and $9.09 \pm 2.04$ with NI-LGA.

Correlations for $\mathrm{V}_{\mathrm{T}}$ and $\mathrm{BP}_{\mathrm{ND}}$ between acquisition durations and between methods were investigated. For LGA, $\mathrm{V}_{\mathrm{T}}(120 \mathrm{~min})$ correlated strongly with $\mathrm{V}_{\mathrm{T}}(180 \mathrm{~min})\left(y=0.99 x-0.46, r^{2}=\right.$ $0.99)$, whereas $\mathrm{BP}_{\mathrm{ND}}(120 \mathrm{~min})$ had a good correlation with $\mathrm{BP}_{\mathrm{ND}}$ (180 min) but with a higher slope $\left(y=1.17 x+0.02, r^{2}=0.99\right)$ because of the $\mathrm{V}_{\mathrm{T}}$ offset ( $y$ intercept of -0.46 ). Both SRTM BP $\mathrm{ND}$ $(120 \mathrm{~min})$ and NI-LGA $\mathrm{BP}_{\mathrm{ND}}(120 \mathrm{~min})$ were in good agreement with 180-min estimates, with a slope of less than $1.09(y=1.07 x+$ $0.00, r^{2}=0.99$, for SRTM and $y=1.09 x+0.01, r^{2}=0.99$, for NI-LGA). There was virtually no $y$ intercept for $\mathrm{BP}_{\mathrm{ND}}(<0.02)$ for the 3 methods. $\mathrm{BP}_{\mathrm{ND}}$ values derived from SRTM or NI-LGA were in good agreement and furthermore agreed with those obtained with the plasma-based LGA method (Fig. 4), in particular for $120 \mathrm{~min}$, with a slope close to 1.0. Test-retest reproducibility for $\mathrm{V}_{\mathrm{T}}$ and $\mathrm{BP}_{\mathrm{ND}}$ is summarized in Supplemental Table 1. Variability of $\mathrm{V}_{\mathrm{T}}$ estimated with LGA was below $13 \%$ and was particularly good in the cerebellum $(\sim 6 \%)$, and variability of $\mathrm{BP}_{\mathrm{ND}}$ was below $10 \%$ for all methods used. Variability was similar between 180- and 120-min acquisitions for both $\mathrm{V}_{\mathrm{T}}$ and $\mathrm{BP}_{\mathrm{ND}}$.

For the occupancy studies, preblocking with tozadenant or preladenant increased the washout in high-uptake regions in a dose-dependent fashion (Figs. 2 and 5) and reduced uptake to levels similar to those in the cerebellum at the highest doses tested, confirming the selectivity of ${ }^{18} \mathrm{~F}$ MNI-444. All analysis methods produced consistent occupancy measurements (Table 2 ), with excellent agreement between occupancies derived using 180 and $120 \mathrm{~min}$ of data, further supporting the reduction of the scanning time to $120 \mathrm{~min}$.

The relationships between the dose of tozadenant or preladenant and $\mathrm{A}_{2 \mathrm{~A}} \mathrm{RO}$ estimated from $120 \mathrm{~min}$ of data are presented 
TABLE 1

${ }^{18} \mathrm{~F}-\mathrm{MNI}-444 \mathrm{~V}_{\mathrm{T}}$ and $\mathrm{BP}_{\mathrm{ND}}$ at Baseline for Acquisitions of 180 Minutes

\begin{tabular}{|c|c|c|c|c|}
\hline Region & $\mathrm{V}_{\mathrm{T}}$ LGA & $\mathrm{BP}_{\mathrm{ND}} \mathrm{LGA}$ & $\mathrm{BP}_{\mathrm{ND}} \mathrm{SRTM}$ & $\mathrm{BP}_{\mathrm{ND}} \mathrm{NI}-\mathrm{LGA}$ \\
\hline Caudate & $21.9 \pm 3.0$ & $5.5 \pm 1.5$ & $6.8 \pm 1.0$ & $6.3 \pm 1.0$ \\
\hline Putamen & $30.3 \pm 5.8$ & $8.0 \pm 2.7$ & $9.6 \pm 1.9$ & $9.1 \pm 2.0$ \\
\hline Globus pallidus & $16.1 \pm 3.9$ & $3.6 \pm 0.7$ & $4.5 \pm 0.9$ & $4.2 \pm 0.9$ \\
\hline Nucleus accumbens & $12.4 \pm 2.1$ & $2.6 \pm 0.4$ & $3.5 \pm 0.4$ & $3.2 \pm 0.3$ \\
\hline Frontal cortex & $4.7 \pm 1.3$ & $0.3 \pm 0.1$ & $0.2 \pm 0.1$ & $0.2 \pm 0.1$ \\
\hline Temporal cortex & $3.5 \pm 0.6$ & $0.0 \pm 0.0$ & $0.0 \pm 0.0$ & $0.0 \pm 0.0$ \\
\hline Occipital cortex & $3.6 \pm 0.3$ & $0.1 \pm 0.1$ & $0.1 \pm 0.1$ & $0.1 \pm 0.1$ \\
\hline Cerebellum & $3.5 \pm 0.7$ & NA & NA & NA \\
\hline \multicolumn{5}{|c|}{$\begin{array}{l}\mathrm{NA}=\text { not applicable. } \\
\text { Data are mean } \pm \text { SD }(n=6)\end{array}$} \\
\hline
\end{tabular}

in Figure 6. Preladenant $\mathrm{ED}_{50}$ was estimated to be $0.013 \pm 0.001$, $0.012 \pm 0.001$, and $0.013 \pm 0.001 \mathrm{mg} / \mathrm{kg}$ with LGA, SRTM, or NILGA, respectively, and tozadenant $\mathrm{ED}_{50}$ was estimated to be $1.44 \pm$ $0.14,1.79 \pm 0.22$, and $1.64 \pm 0.18 \mathrm{mg} / \mathrm{kg}$ with LGA, SRTM, or NILGA, respectively. For 180-min acquisitions, compared with the respective 120-min $\mathrm{ED}_{50}$ estimates, preladenant $\mathrm{ED}_{50}$ was within $20 \%$ for LGA and within $10 \%$ for SRTM and NI-LGA, and tozadenant $\mathrm{ED}_{50}$ was within $5 \%$ for all 3 methods.

The $\mathrm{EC}_{50}$ was estimated from the relationship of the $\mathrm{A}_{2 \mathrm{~A}} \mathrm{RO}$ against the plasma level of tozadenant and preladenant. Estimates were similar for all 3 methods. Using plasma concentrations at the beginning of the scan, LGA $\mathrm{EC}_{50}$ was $877 \pm 109 \mathrm{ng} / \mathrm{mL}$ for tozadenant and $4.0 \pm 0.4 \mathrm{ng} / \mathrm{mL}$ for preladenant, and using average plasma concentrations during the scan, $\mathrm{EC}_{50}$ was $499 \pm 57$ $\mathrm{mg} / \mathrm{mL}$ for tozadenant and $2.1 \pm 0.3 \mathrm{ng} / \mathrm{mL}$ for preladenant. The average of these $2 \mathrm{EC}_{50}$ values for each drug was used for the pharmacokinetic modeling described below: $\mathrm{EC}_{50}=688 \mathrm{ng} / \mathrm{mL}$ for tozadenant and $\mathrm{EC}_{50}=3.1 \mathrm{ng} / \mathrm{mL}$ for preladenant.

\section{Estimated RO in Humans}

In humans, the mean steady-state plasma half-life of tozadenant is $13-15 \mathrm{~h}$, whereas the half-life of preladenant is less than $3 \mathrm{~h}$ for doses of $10 \mathrm{mg}$ and lower (25). The $\mathrm{EC}_{50}$ values in the previous paragraph $(3.1 \mathrm{ng} / \mathrm{mL}$ for preladenant and $688 \mathrm{ng} / \mathrm{mL}$ for tozadenant) were adjusted for differences in free fraction to estimate the $\mathrm{EC}_{50}$ in humans. The free-fraction-adjusted $\mathrm{EC}_{50}$ was $1,147 \mathrm{ng} /$ $\mathrm{mL}$ for tozadenant and $9.2 \mathrm{ng} / \mathrm{mL}$ for preladenant. $\mathrm{A}_{2 \mathrm{~A}} \mathrm{RO}$ over
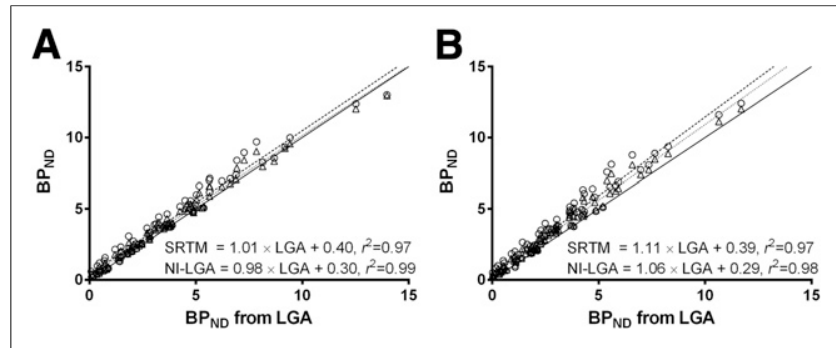

FIGURE 4. Correlation of SRTM and NI-LGA BP ${ }_{N D}$ with LGA BP ${ }_{N D}$ for acquisitions of $120 \mathrm{~min}(\mathrm{~A})$ or $180 \mathrm{~min}(\mathrm{~B})$. $\mathrm{O}=\mathrm{SRTM}$; $\triangle$ = NI-LGA; solid line = line of identity; dashed line = SRTM linear regression fit; dotted line $=$ NI-LGA linear regression fit.
$24 \mathrm{~h}$ was predicted assuming a similar relationship in humans and NHPs between $\mathrm{A}_{2 \mathrm{~A}} \mathrm{RO}$ and free plasma concentrations of tozadenant and preladenant. Occupancy by tozadenant was predicted to be better sustained than that by preladenant (Fig. 7). Preladenant, $10 \mathrm{mg} \mathrm{BID}$, is predicted to achieve approximately $90 \%$ occupancy at $\mathrm{C}_{\max }$ and to drop below $60 \%$ after $6 \mathrm{~h}$. For $180 \mathrm{mg}$ BID of tozadenant, the predicted occupancy at $\mathrm{C}_{\max }$ is approximately $78 \%$ and remains above $70 \%$ until the next dose (12 h).

\section{${ }^{18} \mathrm{~F}-\mathrm{MNI}-444$ Dosimetry}

${ }^{18}$ F-MNI-444 whole-body images are shown in Supplemental Figure 1, and the calculated absorbed doses and whole-body effective doses are presented in Supplemental Table 3. The estimated radiation exposure $(0.022-0.028 \mathrm{mSv} / \mathrm{MBq})$ is in line with other ${ }^{18} \mathrm{~F}$-labeled tracers (e.g., $0.019 \mathrm{mSv} / \mathrm{MBq}$ for ${ }^{18} \mathrm{~F}$-FDG) and would allow several scans to be performed on the same subject with a $180-\mathrm{MBq}$ dose.

\section{DISCUSSION}

The primary aim of this study was to evaluate a new PET radiotracer for in vivo imaging of brain $\mathrm{A}_{2 \mathrm{~A}}$ receptors. The secondary aim was to use pharmacokinetic information from human studies to predict $\mathrm{A}_{2 \mathrm{~A}} \mathrm{RO}$ by tozadenant and preladenant.

Routine production of ${ }^{18} \mathrm{~F}-\mathrm{MNI}-444$ was accomplished using an automated synthesis module. The ${ }^{18} \mathrm{~F}-\mathrm{MNI}-444$ product was purified by reverse-phase high-performance liquid chromatography,

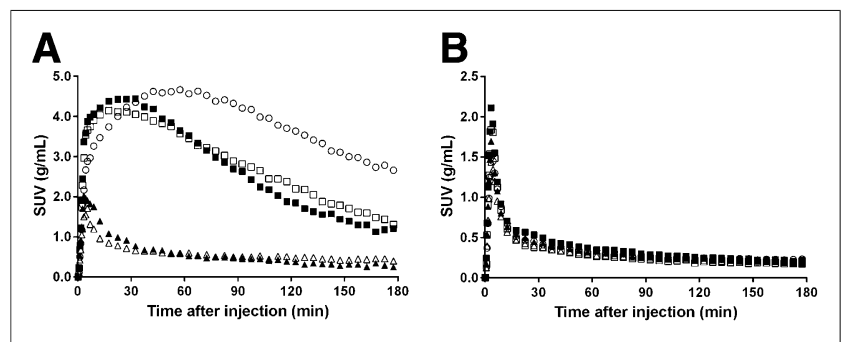

FIGURE 5. Time-activity curves for rhesus macaque in putamen (A) and cerebellum (B) after bolus injection of ${ }^{18} \mathrm{~F}-\mathrm{MNI}-444$ at baseline and after dosing with tozadenant or preladenant. $\mathrm{O}=$ baseline scan; $\mathbf{\square}=$ tozadenant, $1.5 \mathrm{mg} / \mathrm{kg} ; \square=$ preladenant, $0.004 \mathrm{mg} / \mathrm{kg} ; \boldsymbol{\Delta}=$ tozadenant, $10.5 \mathrm{mg} / \mathrm{kg} ; \Delta$ = preladenant, $0.2 \mathrm{mg} / \mathrm{kg}$. 
TABLE 2

Preladenant and Tozadenant $A_{2 A} R O$

\begin{tabular}{|c|c|c|c|c|c|c|c|}
\hline \multirow[b]{2}{*}{ Drug } & \multirow[b]{2}{*}{ Dose (mg/kg) } & \multicolumn{3}{|c|}{ Occupancy (\%) for $180 \mathrm{~min}$ of data } & \multicolumn{3}{|c|}{ Occupancy (\%) for $120 \mathrm{~min}$ of data } \\
\hline & & LGA & SRTM & NI-LGA & LGA & SRTM & NI-LGA \\
\hline \multirow[t]{8}{*}{ Preladenant } & 1.0 & 103 & 100 & 101 & 102 & 100 & 101 \\
\hline & 0.2 & 90 & 92 & 91 & 92 & 93 & 92 \\
\hline & 0.04 & 69 & 76 & 73 & 73 & 78 & 76 \\
\hline & 0.015 & 39 & 45 & 42 & 45 & 49 & 45 \\
\hline & 0.015 & 53 & 56 & 55 & 52 & 58 & 56 \\
\hline & 0.010 & 35 & 44 & 40 & 43 & 49 & 45 \\
\hline & 0.004 & 32 & 26 & 27 & 32 & 27 & 27 \\
\hline & 0.004 & 23 & 25 & 25 & 29 & 26 & 27 \\
\hline \multirow[t]{6}{*}{ Tozadenant } & 10.5 & 95 & 94 & 94 & 94 & 94 & 94 \\
\hline & 7.5 & 93 & 84 & 88 & 91 & 83 & 87 \\
\hline & 5.0 & 76 & 68 & 71 & 76 & 66 & 69 \\
\hline & 1.5 & 47 & 42 & 44 & 49 & 42 & 44 \\
\hline & 1.3 & 43 & 44 & 46 & 44 & 43 & 45 \\
\hline & 0.5 & 28 & 26 & 27 & 26 & 26 & 25 \\
\hline
\end{tabular}

formulated into a physiologic solution, and filtered into a sterile final product vial in a total production time of less than $60 \mathrm{~min}$ with an average decay-corrected yield of around 25\%, a radiochemical purity greater than $99 \%$, and a specific activity in excess of $370 \mathrm{GBq} / \mu \mathrm{mol}$.

${ }^{18} \mathrm{~F}-\mathrm{MNI}-444$ demonstrated high brain uptake in rhesus monkeys and a distribution in agreement with the known distribution

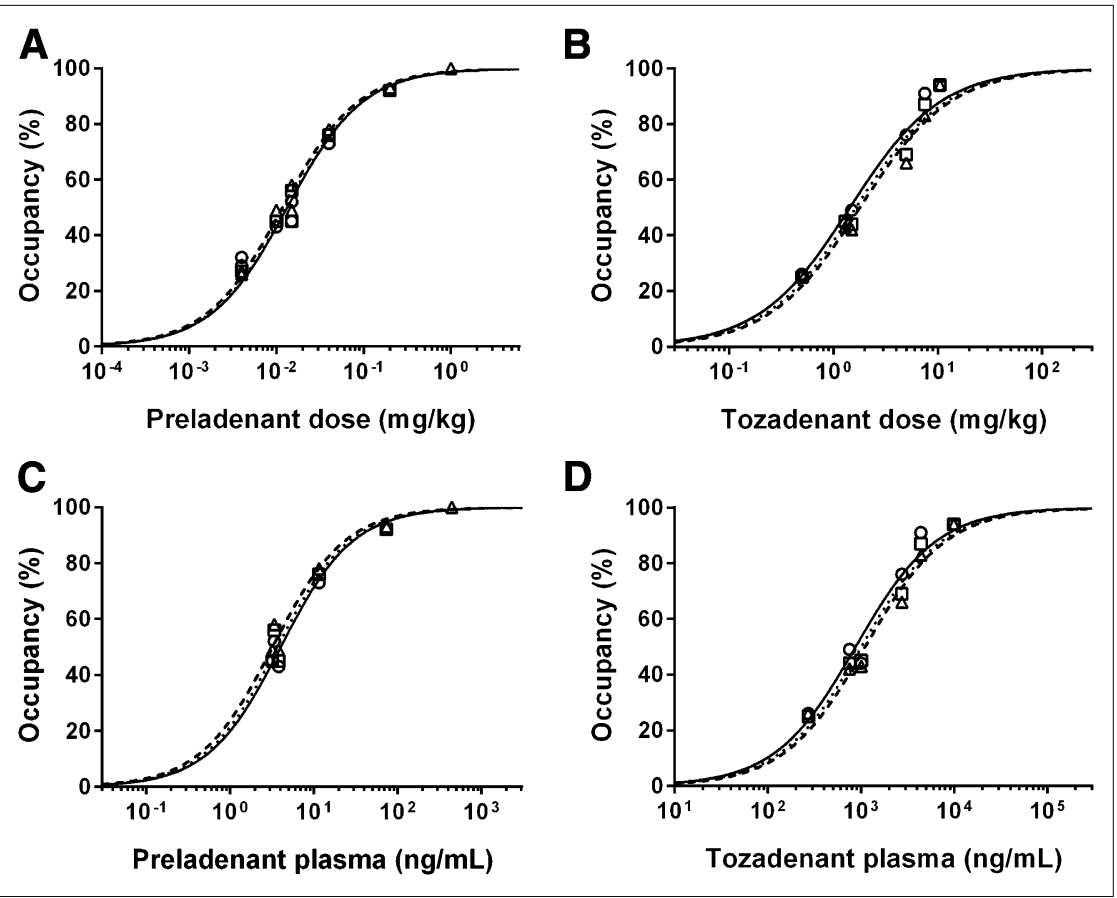

FIGURE 6. Striatal $A_{2 A} R O$ against preladenant $(A)$ or tozadenant $(B)$ administered doses, and against preladenant $(C)$ or tozadenant $(D)$ plasma levels at beginning of scan. Symbols represent occupancy estimates using LGA $(O)$, SRTM $(\triangle)$, or NI-LGA $(\square)$ for $120 \mathrm{~min}$ of data. Lines represent model fits using LGA (solid line), SRTM (dashed line), or NI-LGA (dotted lines) occupancy estimates. of $\mathrm{A}_{2 \mathrm{~A}}$ receptor. ${ }^{18} \mathrm{~F}-\mathrm{MNI}-444$ had a moderate metabolism rate, unaffected by preinjection of the 2 selective $\mathrm{A}_{2 \mathrm{~A}}$ antagonists tozadenant and preladenant.

Time-activity curves were also analyzed using standard 1- and 2-tissue-compartment models (data not shown) (26). The 1-tissuecompartment model did not provide good fits visually in any regions and gave higher Akaike criteria than the 2-tissuecompartment model. On the other hand, the 2-tissue-compartment model adequately described curves in high-uptake regions but had convergence issues in cortical regions and in the cerebellum, with a close-to-zero $k_{4}$ rate constant (23). Therefore 1- and 2-tissuecompartment models were not investigated further.

The relationship between $\mathrm{V}_{\mathrm{T}}(120 \mathrm{~min})$ and $\mathrm{V}_{\mathrm{T}}(180 \mathrm{~min})$ was close to the line of identity for LGA, with a small offset. In high-uptake regions, a 120-min acquisition resulted in a slightly smaller $(<5 \%)$ $\mathrm{V}_{\mathrm{T}}$, whereas in the cerebellum the difference was larger $(-17 \%)$. For $\mathrm{BP}_{\mathrm{ND}}$, a similar effect was observed, with an acceptable difference of less than $10 \%$ for the reference region-based methods and a good correlation of $\mathrm{BP}_{\mathrm{ND}}$ among all 3 methods. For SRTM, the improvement in the fits for shorter acquisitions could be due to a more 1-tissue-compartment model-like signal or to a reduction of the effects of an unlikely but potentially slowly penetrating radiometabolite. For the occupancy estimates, there was excellent agreement for all methods and acquisition durations, with the test-retest reproducibility remaining excellent for acquisitions of $2 \mathrm{~h}$. 


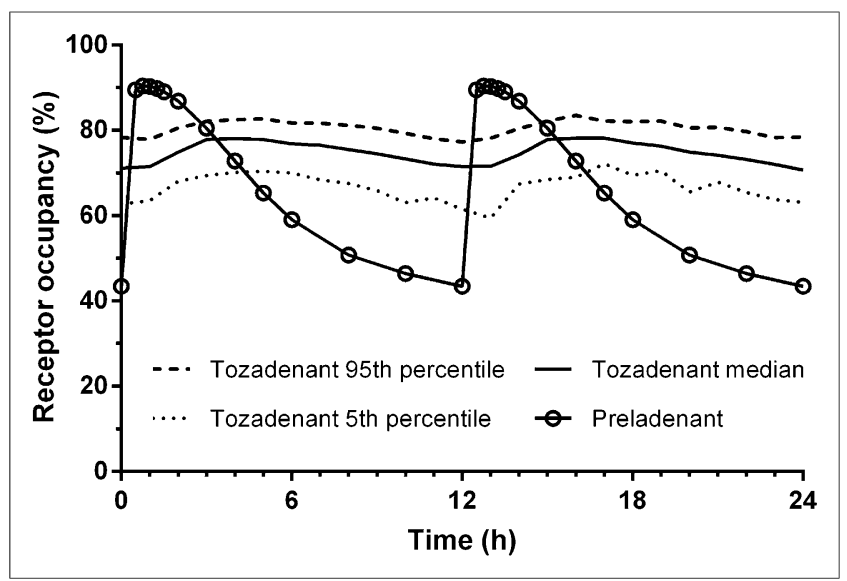

FIGURE 7. Pharmacokinetic modeling of tozadenant and preladenant plasma concentrations in humans was used to predict $A_{2 A} R O$ after repeated dosing (180 mg BID of tozadenant and $10 \mathrm{mg}$ BID of preladenant).

Pharmacokinetic modeling of human plasma levels of tozadenant and preladenant combined with corrected NHP $\mathrm{EC}_{50}$ estimates suggested that the median $\mathrm{A}_{2 \mathrm{~A}} \mathrm{RO}$ of $180-\mathrm{mg}-$ BID tozadenant will be sustained, whereas the RO of $10-\mathrm{mg}-$ BID preladenant will drop below $60 \%$ after $6 \mathrm{~h}$. Importantly, these predictions assume that the $\mathrm{EC}_{50}$ is the same in rhesus monkeys and humans and could be substantially different from those estimated here should that not be so. The potential clinical relevance of this difference in RO remains to be determined.

A dose-independent reduction of $\mathrm{V}_{\mathrm{T}}$ in the cerebellum of less than $15 \%$ was observed for all preblocking studies compared with baseline. The cerebellum has low to negligible $\mathrm{A}_{2 \mathrm{~A}}$ receptor density (9), and this region was used as a reference in previous studies with other $\mathrm{A}_{2 \mathrm{~A}}$ radiotracers $(5,8)$. It is therefore unclear whether this reduction is due to blocking of specific binding in the cerebellum (unlikely because of the dose independence) or other factors such as small changes in the plasma protein binding that would be difficult to detect given the low free fraction $(\sim 1 \%)$. However, if one conservatively assumes a specific signal in the cerebellum of $20 \%$ of the total signal, occupancy measurement error (maximum, $\sim 50 \%$ occupancy) would be less than 5\% (27, 28). The excellent agreement in the occupancy estimates between the plasma-based and reference region-based methods suggests that $\mathrm{A}_{2 \mathrm{~A}}$ occupancy can be quantitatively assessed in rhesus monkeys using SRTM or NI-LGA without the need for arterial sampling.

With a maximum striatum-to-cerebellum ratio of about 12.0 at $60-70 \mathrm{~min},{ }^{18} \mathrm{~F}-\mathrm{MNI}-444$ appears superior to other $\mathrm{A}_{2 \mathrm{~A}}$ PET tracers such as ${ }^{11} \mathrm{C}$-TMSX and ${ }^{11} \mathrm{C}-\mathrm{SCH} 442416$, with a ratio of about 1.5 at $60 \mathrm{~min}$ being measured for ${ }^{11} \mathrm{C}$-TMSX (previously denoted $\left.{ }^{11} \mathrm{C}-\mathrm{KF} 18446\right)$ in rhesus monkeys (8) and a ratio of about 2.2 at $15 \mathrm{~min}$ being measured for ${ }^{11} \mathrm{C}$-SCH442416 in Macaca nemestrina (5).

\section{CONCLUSION}

We report here the evaluation of ${ }^{18} \mathrm{~F}-\mathrm{MNI}-444$ in nonhuman primates. ${ }^{18} \mathrm{~F}-\mathrm{MNI}-444$ had regional uptake consistent with $\mathrm{A}_{2 \mathrm{~A}}$ receptor distribution and with much improved binding ratios com- pared with currently available $\mathrm{A}_{2 \mathrm{~A}}$ PET radiotracers. The selectivity of ${ }^{18} \mathrm{~F}-\mathrm{MNI}-444$ for $\mathrm{A}_{2 \mathrm{~A}}$ was demonstrated against $2 \mathrm{~A}_{2 \mathrm{~A}}$ antagonists. Noninvasive quantification of ${ }^{18} \mathrm{~F}-\mathrm{MNI}-444$ with SRTM or LGA using the cerebellum as a reference is possible for 120-min acquisitions, in particular for occupancy studies. ${ }^{18} \mathrm{~F}$ MNI-444 dosimetry is favorable, with an effective dose consistent with values reported for other PET radiotracers used in humans. Therefore, ${ }^{18} \mathrm{~F}-\mathrm{MNI}-444$ has great potential as a PET radiotracer for $\mathrm{A}_{2 \mathrm{~A}}$ receptor imaging in humans. Lastly, because of differences in pharmacokinetics in humans, $\mathrm{A}_{2 \mathrm{~A}} \mathrm{RO}$ by tozadenant is predicted to be more sustained than $\mathrm{A}_{2 \mathrm{~A}} \mathrm{RO}$ by preladenant at clinical doses.

\section{DISCLOSURE}

The costs of publication of this article were defrayed in part by the payment of page charges. Therefore, and solely to indicate this fact, this article is hereby marked "advertisement" in accordance with 18 USC section 1734. This study was financed by UCB Biopharma SPRL. No other potential conflict of interest relevant to this article was reported.

\section{REFERENCES}

1. Wright Willis A, Evanoff BA, Lian M, Criswell SR, Racette BA. Geographic and ethnic variation in Parkinson disease: a population-based study of US Medicare beneficiaries. Neuroepidemiology. 2010;34:143-151.

2. Driver JA, Logroscino G, Gaziano JM, Kurth T. Incidence and remaining lifetime risk of Parkinson disease in advanced age. Neurology. 2009;72:432438.

3. Scherman D, Desnos C, Darchen F, Pollak P, Javoy-Agid F, Agid Y. Striatal dopamine deficiency in Parkinson's disease: role of aging. Ann Neurol. 1989;26: $551-557$.

4. Greffard S, Verny M, Bonnet AM, et al. Motor score of the Unified Parkinson Disease Rating Scale as a good predictor of Lewy body-associated neuronal loss in the substantia nigra. Arch Neurol. 2006;63:584-588.

5. Moresco RM, Todde S, Belloli S, et al. In vivo imaging of adenosine A2A receptors in rat and primate brain using $\left[{ }^{11} \mathrm{C}\right] \mathrm{SCH} 442416$. Eur J Nucl Med Mol Imaging. 2005;32:405-413.

6. Hirani E, Gillies J, Karasawa A, et al. Evaluation of [4-O-methyl- $\left.{ }^{11} \mathrm{C}\right] \mathrm{KW}-6002$ as a potential PET ligand for mapping central adenosine $\mathrm{A}_{2 \mathrm{~A}}$ receptors in rats. Synapse. 2001;42:164-176.

7. Svenningsson P, Hall H, Sedvall G, Fredholm BB. Distribution of adenosine receptors in the postmortem human brain: an extended autoradiographic study. Synapse. 1997;27:322-335.

8. Ishiwata K, Noguchi J, Wakabayashi S, et al. ${ }^{11} \mathrm{C}$-labeled KF18446: a potential central nervous system adenosine A2a receptor ligand. J Nucl Med. 2000;41: 345-354.

9. Sihver W, Schulze A, Wutz W, et al. Autoradiographic comparison of in vitro binding characteristics of various tritiated adenosine A2A receptor ligands in rat, mouse and pig brain and first ex vivo results. Eur J Pharmacol. 2009;616:107114.

10. Stone TW, Ceruti S, Abbracchio MP. Adenosine receptors and neurological disease: neuroprotection and neurodegeneration. Handb Exp Pharmacol. 2009: 535-587.

11. Rissanen E, Virta JR, Paavilainen T, et al. Adenosine A2A receptors in secondary progressive multiple sclerosis: a $\left[{ }^{11} \mathrm{C}\right] \mathrm{TMSX}$ brain PET study. J Cereb Blood Flow Metab. 2013;33:1394-1401.

12. Holschbach MH, Bier D, Stusgen S, et al. Synthesis and evaluation of 7-amino2-(2(3)-furyl)-5-phenylethylamino-oxazolo[5,4-d]pyrimidines as potential A2A adenosine receptor antagonists for positron emission tomography (PET). Eur J Med Chem. 2006;41:7-15.

13. Ferre S, von Euler G, Johansson B, Fredholm BB, Fuxe K. Stimulation of high-affinity adenosine A2 receptors decreases the affinity of dopamine D2 receptors in rat striatal membranes. Proc Natl Acad Sci USA. 1991;88:72387241

14. LeWitt PA, Guttman M, Tetrud JW, et al. Adenosine A2A receptor antagonist istradefylline (KW-6002) reduces "off" time in Parkinson's disease: a doubleblind, randomized, multicenter clinical trial (6002-US-005). Ann Neurol. 2008; 63:295-302. 
15. Hauser RA, Cantillon M, Pourcher E, et al. Preladenant in patients with Parkinson's disease and motor fluctuations: a phase 2, double-blind, randomised trial. Lancet Neurol. 2011;10:221-229.

16. Hauser RA, Olanow CW, Kieburtz K, et al. A phase 2, placebo-controlled, randomized, double-blind trial of tozadenant (SYN-115) in patients with Parkinson's disease with wearing-off fluctuations on levodopa [abstract]. Mov Disord. 2013;28 (suppl 1):444.

17. Dungo R, Deeks ED. Istradefylline: first global approval. Drugs. 2013;73:875-882.

18. Stone-Elander S, Thorell JO, Eriksson L, Fredholm BB, Ingvar M. In vivo biodistribution of $\left[\mathrm{N}-{ }^{11} \mathrm{C}\right.$-methyl]KF 17837 using 3-D-PET: evaluation as a ligand for the study of adenosine A2A receptors. Nucl Med Biol. 1997;24:187-191.

19. Noguchi J, Ishiwata K, Wakabayashi S, et al. Evaluation of carbon-11-labeled KF17837: a potential CNS adenosine A2a receptor ligand. J Nucl Med. 1998;39: 498-503.

20. Bhattacharjee AK, Lang L, Jacobson O, et al. Striatal adenosine $\mathrm{A}_{2 \mathrm{~A}}$ receptormediated positron emission tomographic imaging in 6-hydroxydopaminelesioned rats using [ $\left.{ }^{18} \mathrm{~F}\right]-$ MRS5425. Nucl Med Biol. 2011;38:897-906.

21. Tavares AA, Batis J, Barret O, et al. In vivo evaluation of $\left[{ }^{123} \mathrm{I}\right] \mathrm{MNI}-420$ : a novel single photon emission computed tomography radiotracer for imaging of adenosine 2A receptors in brain. Nucl Med Biol. 2013;40:403-409.
22. Logan J. A review of graphical methods for tracer studies and strategies to reduce bias. Nucl Med Biol. 2003;30:833-844.

23. Innis RB, Cunningham VJ, Delforge J, et al. Consensus nomenclature for in vivo imaging of reversibly binding radioligands. J Cereb Blood Flow Metab. 2007; 27:1533-1539.

24. Lammertsma AA, Hume SP. Simplified reference tissue model for PET receptor studies. Neuroimage. 1996;4:153-158.

25. Cutler DL, Tendolkar A, Grachev ID. Safety, tolerability and pharmacokinetics after single and multiple doses of preladenant (SCH420814) administered in healthy subjects. J Clin Pharm Ther. 2012;37:578-587.

26. Slifstein M, Laruelle M. Models and methods for derivation of in vivo neuroreceptor parameters with PET and SPECT reversible radiotracers. Nucl Med Biol. 2001;28:595-608.

27. Gunn RN, Murthy V, Catafau AM, et al. Translational characterization of $\left[{ }^{11} \mathrm{C}\right]$ GSK931145, a PET ligand for the glycine transporter type 1. Synapse. 2011;65: 1319-1332.

28. Turkheimer FE, Selvaraj S, Hinz R, et al. Quantification of ligand PET studies using a reference region with a displaceable fraction: application to occupancy studies with $\left[{ }^{11} \mathrm{C}\right]-\mathrm{DASB}$ as an example. J Cereb Blood Flow Metab. 2012;32: $70-80$. 